\title{
Use of Lateral Oncoplasty for Multiquadrant Giant Fibroadenoma: A Novel Approach
}

\author{
Sanghamitra Jena ${ }^{1}$, Neetesh K. Sinha ${ }^{2}$ \\ IND \\ Corresponding author: Sanghamitra Jena, docsalu@gmail.com
}

1. Surgical Oncology, Tata Main Hospital, Jamshedpur, IND 2. Surgical Oncology, Medica Cancer Hospital, Rangapani,

\begin{abstract}
Giant fibroadenomas are uncommon benign lesions, defined as fibroadenomas of $>5 \mathrm{~cm}$ in size and/or weighing more than $500 \mathrm{~g}$. They can distort the shape of the breast and cause asymmetry, so they should be excised. Here, we report two cases of giant fibroadenoma, where wide local excision and reconstruction with lateral oncoplasty were done. Compared to all previous reports of patients with giant fibroadenoma, where the lump was excised either through a submammary incision or by round block technique depending on the location of the tumour, we used the lateral oncoplasty technique in both patients. Lateral oncoplasty is a new reconstructive option to maintain cosmesis and symmetry after the excision of giant fibroadenomas in the outer and central quadrants of the breast. It is a good option for reconstruction in cases where the defect is very large and facilities for conventional flap surgeries are not available.
\end{abstract}

Categories: General Surgery

Keywords: giant fibroadenoma, breast, lateral oncoplasty, reconstruction, cosmesis

\section{Introduction}

Fibroadenomas in the breast are common benign lesions in women less than 30 years of age. They feature polyclonal proliferation of both epithelial and stromal tissue and are hyperplastic lesions rather than true neoplasms [1]. Giant fibroadenomas, defined as fibroadenomas of greater than $5 \mathrm{~cm}$ in size or $500 \mathrm{~g}$ in weight, are rare benign breast lesions that account for approximately $0.5 \%-2 \%$ of fibroadenomas [2-4]. We report two cases of giant fibroadenoma treated with a new technique of excision and reconstruction with lateral oncoplasty.

\section{Case Presentation}

\section{Case 1}

A 25 -year-old woman presented with a right breast lump existing for eight months. The mass had rapidly increased in size. Her menstrual cycles were regular and she was not on any hormone therapy. Clinical examination showed a firm and mobile breast tumour on the outer quadrant of the right breast of

Review began 05/01/2021 Review ended 05/18/2021 Published 05/18/2021

\section{(c) Copyright 2021}

Jena et al. This is an open access article distributed under the terms of the Creative Commons Attribution License CC-BY 4.0., which permits unrestricted use, distribution, and reproduction in any medium, provided the original author and source are credited. approximately $10 \mathrm{~cm}$ in size, with dilated veins on overlying skin (Figures $1 \mathrm{~A}, 2$ ). There was no axillary lymphadenopathy. Ultrasonography (US) showed a well-defined hypoechoic tumour measuring $10 \mathrm{x} 8 \mathrm{~cm}$. Core needle biopsy confirmed fibroadenoma. 


\section{Cureus}

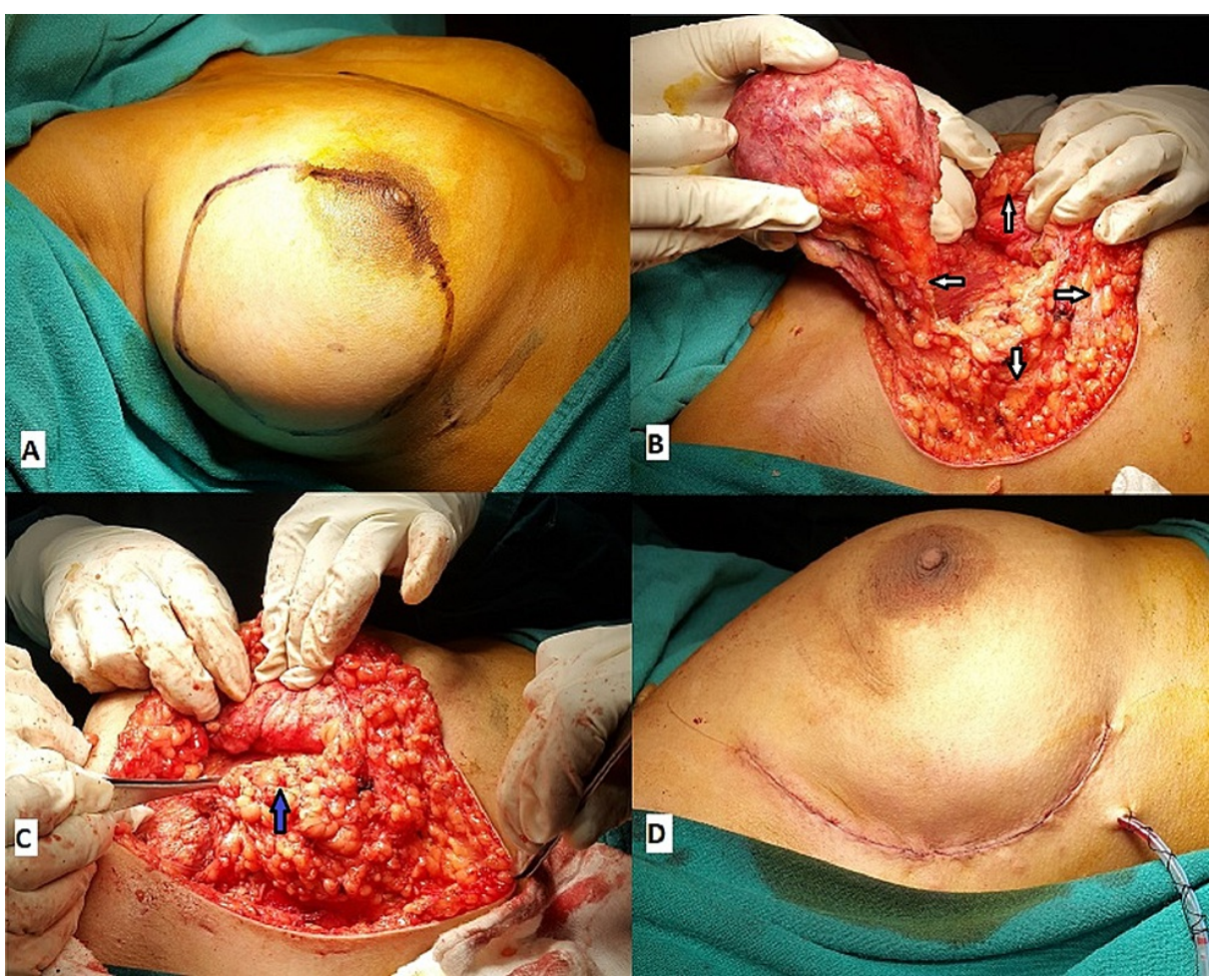

FIGURE 1: A. Giant fibroadenoma in the outer quadrant of the right breast. B. Well-defined lump excised in total (defect area marked with white arrows). C. Infero-lateral glandular flap raised (marked with blue arrow) and advanced upward and medially. D. Wound closed with subcuticular sutures.

Case 1

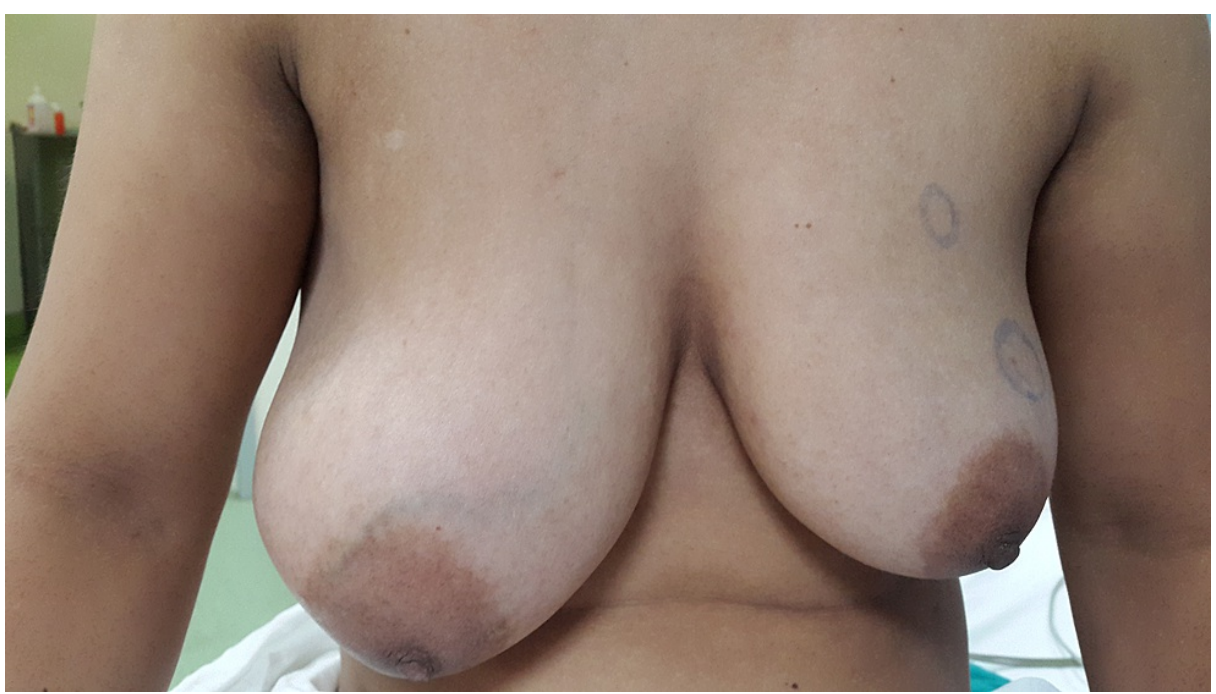

FIGURE 2: Pre-operative picture of Case 1.

The patient underwent right-sided lumpectomy and reconstruction with lateral oncoplasty under general anaesthesia. A skin crease incision was placed in the axillary fold. Subsequently, the skin flap was raised medially over the tumour with a flap thickness of 3-4 mm. The lump was well defined (Figure 1B) and almost the entire normal breast tissue of the affected breast was compressed on to one side by the lump. The lump was completely excised, which left a big defect. An inferolateral glandular flap was raised and advanced upward and medially and fixed with a $2 / 0$ absorbable monofilament suture to the compressed normal breast 


\section{Cureus}

parenchyma (Figure 1C). A suction drain was left in situ over the pectoral fascia and the wound closed with subcuticular sutures (Figure 1D).

The post-operative period was uneventful. The patient was discharged on the third postoperative day after the removal of the drain. The patient was followed up at 10 days, one month, three months, and one year. Complete symmetry was achieved between both the breasts with good cosmesis (Figure 3).

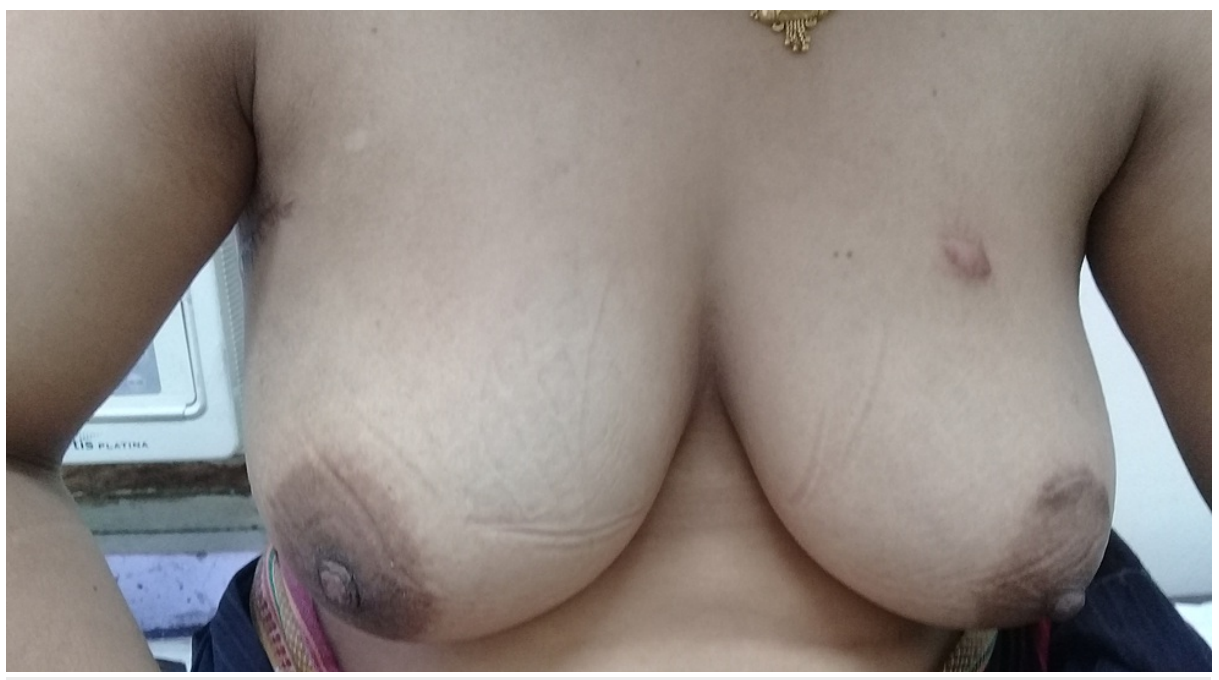

FIGURE 3: Post operative picture of Case 1 after three months.

The actual size of the tumour was $10 \times 8 \times 4 \mathrm{~cm}$. Histopathology showed marked stromal and epithelial proliferation with proliferating stroma around numerous ducts in a peri-canalicular pattern. There was no stromal atypia and no evidence of malignancy. The final diagnosis was reported as giant fibroadenoma.

\section{Case 2}

A 28-year-old woman presented with a left breast lump existing for one year. The mass had rapidly increased in size. Clinical examination showed a firm lump in the central quadrant of the left breast of approximately $12 \mathrm{~cm}$ in size, with dilated veins on overlying skin (Figure 4). There was no axillary lymphadenopathy. Ultrasonography showed a large well-defined hypoechoic lobulated mass lesion with homogenous echo pattern and internal vascularity in the left-central quadrant measuring $13.8 \times 10.5 \mathrm{~cm}$. Core needle biopsy findings were compatible with benign fibro-epithelial lesion favouring benign phyllodes tumour.

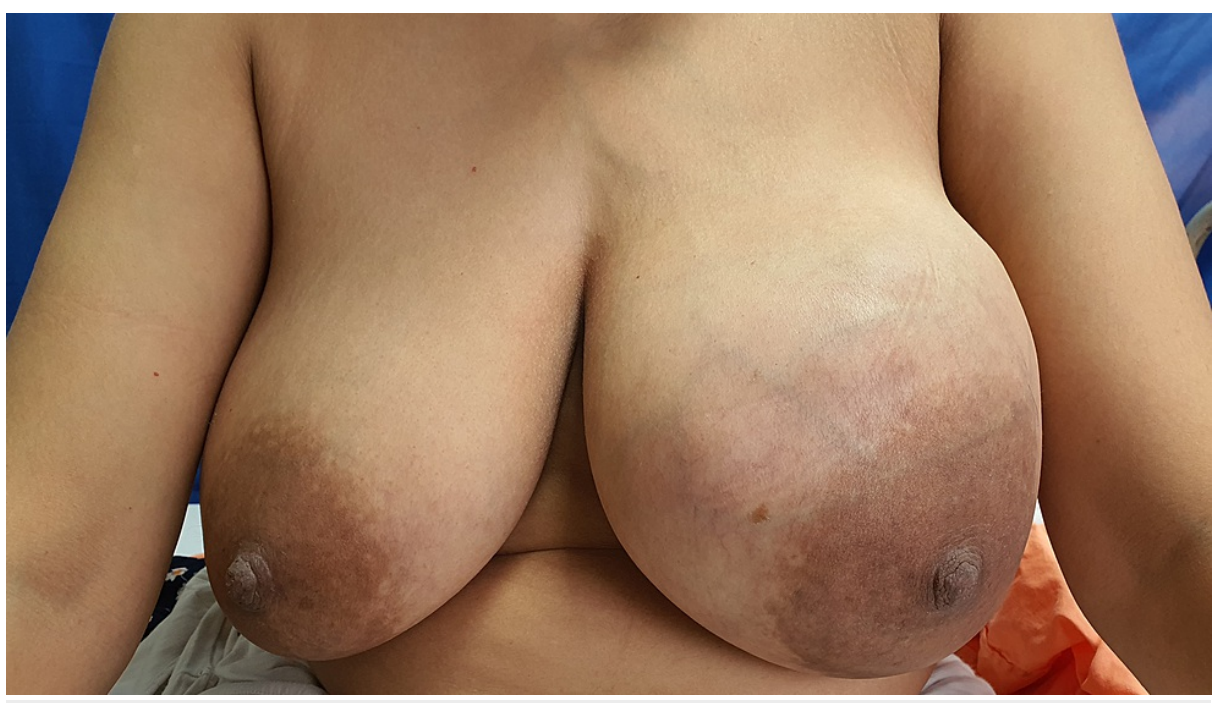

FIGURE 4: Pre- operative picture of Case 2.

The patient was planned for wide local excision of left breast lump and reconstruction with lateral 


\section{Cureus}

oncoplasty. A skin crease incision was made in lateral axillary fold and flap was raised over the tumour with a thickness of 3-4 mm (Figure 5A). The lump was excised with a margin of $1 \mathrm{~cm}$ (Figures $5 B, 5 C$ ). An inferolateral pedicled dermo-glandular flap was raised and rotated upwards and medially to cover the defect (Figure 5D). A suction drain was placed in situ and wound closed with subcuticular stitches. The drain was removed on the third postoperative day and the patient followed up in OPD on the 10th day, one month, and three months. There was no recurrence, and a good cosmesis was achieved (Figure 6).

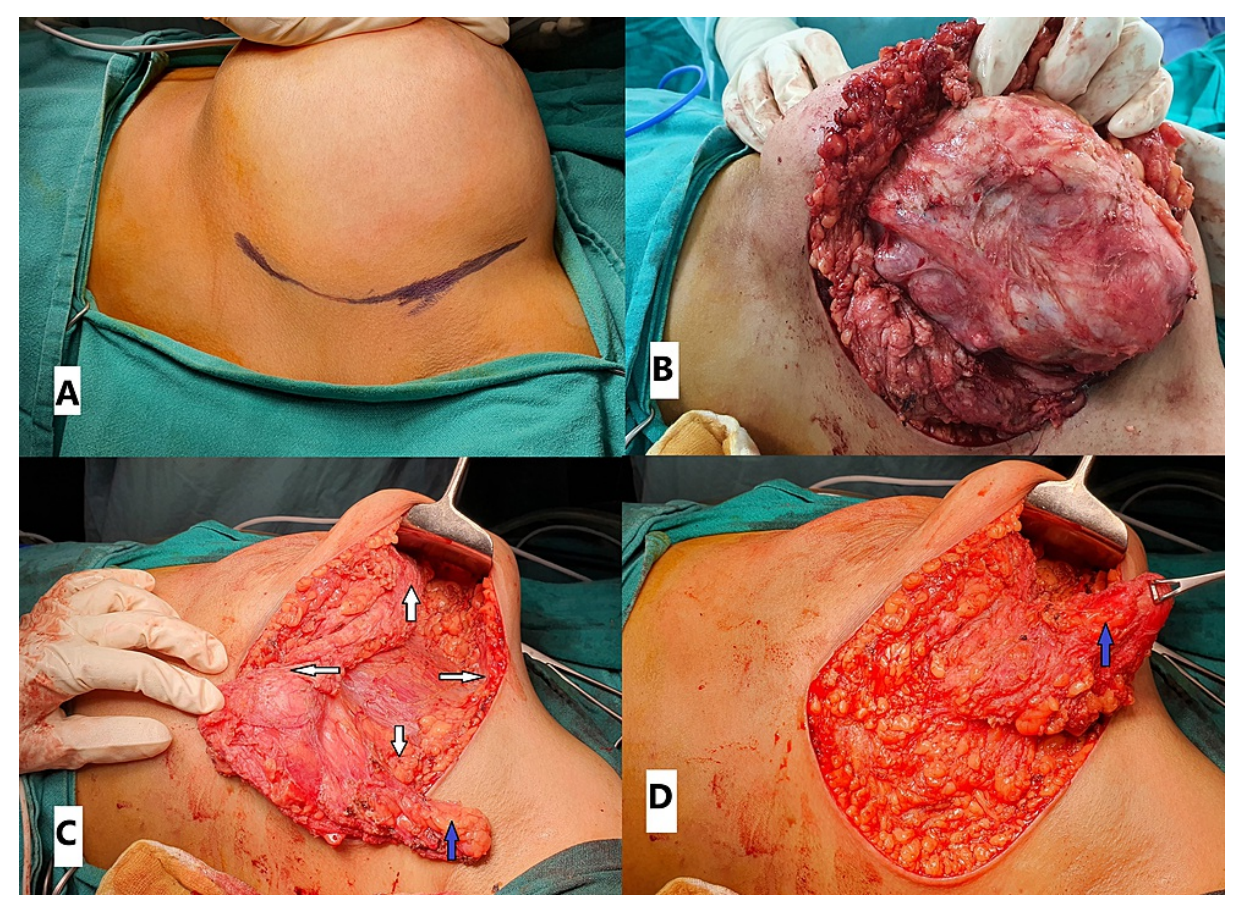

FIGURE 5: A. Skin crease incision given in lateral axillary fold. B. Welldefined lump. C. Lump excised in total (defect area marked with white arrow and flap marked with blue arrow). D. Lateral oncoplasty done (flap marked with blue arrow).

Case 2.

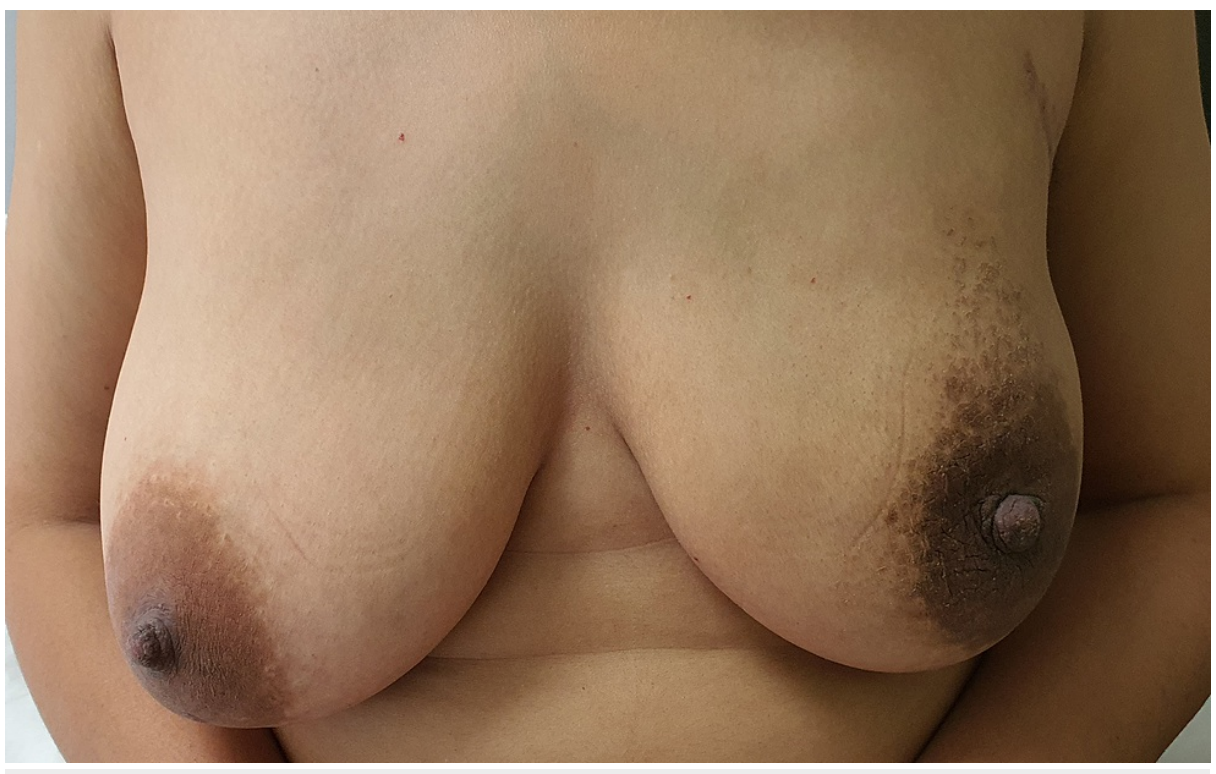

FIGURE 6: Post operative picture of Case 2 after three months.

The tumour measured $15.5 \times 14.5 \times 9 \mathrm{~cm}$ and weighed $843 \mathrm{~g}$. On cross-section, the lesion was bosselated, 


\section{Discussion}

Giant fibroadenomas are defined as fibroadenomas of greater than $5 \mathrm{~cm}$ in size or $500 \mathrm{~g}$ in weight. They are rare benign breast lesions and account for approximately $0.5 \%-2 \%$ of fibroadenomas [2-4]. Both the cases fit well into the criteria of giant fibroadenomas as they are more than $5 \mathrm{~cm}$ and weighing more than $500 \mathrm{~g}$. The peak age has been reported between 17 and 20 years [5]. Excessive estrogen stimulation and/or receptor sensitivity or reduced levels of estrogen antagonist during puberty have been implicated in pathogenesis $[6,7]$.

There are a number of differential diagnoses of giant fibroadenomas. In the second case, it was confused with phyllodes tumour. In fact, the clinical and sonographic appearance of giant fibroadenoma is indistinguishable from phyllodes tumour. The characteristics of fibroadenoma include well-demarcated margins, minimal atypia, and rare mitosis while phyllodes tumours sometimes show invasive margins, matrix overgrowth and significant atypia, and a leafy structure [8,9]. Fibroadenomas are frequently hypoechoic with circumscribed margins on US while phyllodes tumors are irregular in shape with heterogeneous internal echogenicity and microlobulated margins. Because the examination of small samples from needle biopsy may yield uncertain results, the final diagnosis is made based on the histopathological report. In the second case, the pathological diagnosis of giant fibroadenoma was made based on the examination of the surgical specimen.

Rapid growth, distortion of breast architecture or with overlying skin changes, and risk of malignancy are indications for surgical excision of a suspected fibroadenoma [4,10]. The goals of surgical reconstruction for giant fibroadenomas with asymmetry should be to achieve equal breast size, reposition the nipple-areola complex, and correct asymmetry with minimal scars in one stage. Multiple treatment modalities ranging from simple excision to mastectomy have been described in the literature [11-13]. The most common incisions reported for excision of giant fibroadenomas are inframammary skin incision, reduction mammaplasty incision, and peri-/circum-areolar incision [1,11,14,15].

In both these cases, a new technique of lateral oncoplasty was used to fill the large defect created after the excision of giant fibroadenoma. This surgical technique was first described for biopsy-proven breast cancer or phyllodes tumour present in the outer quadrant of the breast by Singh et al. [16]. But in our cases, the technique of lateral oncoplasty was used for the reconstruction of tumour involving both outer and central quadrants. In this technique, the breast was dissected of the pectoralis major well beyond the tumor margins. The anterior surface of the breast was separated from the skin, in a plane similar to that while performing mastectomy. Thus, a biplanar mobilization was achieved. The inferolateral glandular flap raised from the inferior quadrant of the breast and lateral axillary fold was then advanced and rotated upward and medially and fixed with $2 / 0$ absorbable monofilament suture with remaining compressed normal breast parenchyma. Here, the upper flap was not needed to achieve a good breast mound and symmetry, as described in Singh et al.'s technique [16].

We believe that this approach offers several distinct advantages. There is a single scar in the lateral aspect of the chest wall which remains hidden in the lateral axillary fold. This gives a superior aesthetic result by avoiding any scar over the breast itself. Larger tumors can be excised using this approach as is evident from the size of the tumor and the weight of the resected specimens in both patients. This technique has reduced the use of latissimus dorsi mini flaps for volume replacement in our hands. Therefore, this is not only a more straightforward technique than the current conventional flaps but also a technique with short operation time and less postoperative pain or other morbidities to patients.

The complications associated with this technique are surgical site infections, delayed wound healing, marginal skin necrosis, superficial NAC (nipple-areola complex) necrosis, and fat necrosis [16]. But in our cases, there was no complication postoperatively. The surgical site infection which occurs most commonly can be managed according to the American Society of Breast Surgeons consensus guideline on preoperative antibiotics and surgical site infections (SSI) in breast surgery [17]. The skin margin necrosis and NAC necrosis can be managed surgically by debridement and margin revision. The fat necrosis can be managed conservatively or with debridement.

\section{Conclusions}

Giant fibroadenoma causes asymmetry in both breasts and requires wide local excision. This causes a large defect after excision. Lateral oncoplasty is a new reconstructive option to maintain cosmesis and symmetry after the excision of giant fibroadenomas in the outer and central quadrants of the breast. This procedure may be worth considering as an option for reconstruction after the excision of giant fibroadenoma in cases where the defect is large and facilities for conventional flap surgeries are not available.

\section{Additional Information}

Disclosures 
Human subjects: Consent was obtained or waived by all participants in this study. Conflicts of interest: In compliance with the ICMJE uniform disclosure form, all authors declare the following: Payment/services info: All authors have declared that no financial support was received from any organization for the submitted work. Financial relationships: All authors have declared that they have no financial relationships at present or within the previous three years with any organizations that might have an interest in the submitted work. Other relationships: All authors have declared that there are no other relationships or activities that could appear to have influenced the submitted work.

\section{References}

1. Park CA, David LR, Argenta LC: Breast asymmetry: presentation of a giant fibroadenoma . Breast J. 2006, 12:451-61. 10.1111/j.1075-122X.2006.00303.X

2. Greenberg R, Skornick Y, Kaplan O: Management of breast fibroadenomas. J Gen Intern Med. 1998, 13:6405. 10.1046/j.1525-1497.1998.cr188.x

3. Sosin M, Pulcrano M, Feldman ED, Patel KM, Nahabedian MY, Weissler JM, Rodriguez ED: Giant juvenile fibroadenoma: a systematic review with diagnostic and treatment recommendations. Gland Surg. 2015, 4:312-21. 10.3978/j.issn.2227-684X.2015.06.04

4. Jayasinghe Y, Simmons PS: Fibroadenomas in adolescence. Curr Opin Obstet Gynecol. 2009, 21:402-6. 10.1097/GCO.0b013e32832fa06b

5. Kupsik M, Yep B, Sulo S, Memmel H: Giant juvenile fibroadenoma in a 9-year-old: a case presentation and review of the current literature. Breast Dis. 2017, 37:95-8. 10.3233/BD-160250

6. Gaurav K, Chandra G, Neelam K, Kumar S, Singla H, Yadav SK: A pre-pubertal girl with giant juvenile fibroadenoma: a rare case report. Int J Surg Case Rep. 2015, 16:87-9. 10.1016/j.ijscr.2015.09.026

7. Ferdousee I, Arafat SM, Ahmed Z: An adolescent girl with giant fibroadenoma - a case report . Pol Przegl Chir. 2016, 88:218-20. 10.1515/pjs-2016-0055

8. Krishnamurthy S, Ashfaq R, Shin HJ, Sneige N: Distinction of phyllodes tumor from fibroadenoma: a reappraisal of an old problem. Cancer. 2000, 90:342-9.

9. Lee AH, Hodi Z, Ellis IO, Elston CW: Histological features useful in the distinction of phyllodes tumour and fibroadenoma on needle core biopsy of the breast. Histopathology. 2007, 51:336-44. 10.1111/j.13652559.2007.02786.x

10. Cerrato FE, Pruthi S, Boughey JC, et al.: Intermediate and long-term outcomes of giant fibroadenoma excision in adolescent and young adult patients. Breast J. 2015, 21:254-9. 10.1111/tbj.12394

11. Ugburo AO, Olajide TO, Fadeyibi IO, Mofikoya BO, Lawal AO, Osinowo AO: Differential diagnosis and management of giant fibroadenoma: comparing excision with reduction mammoplasty incision and excision with inframammary incision. J Plast Surg Hand Surg. 2012, 46:354-8. 10.3109/2000656X.2012.697066

12. Sosin M, Feldman E: Giant juvenile fibroadenoma: a case and review of novel modalities in treatment . Breast Dis. 2012, 34:35-8. 10.3233/BD-130342

13. Meng X, Yamanouchi K, Kuba S, et al.: Giant fibroadenoma of the breast: a rare case in a mature woman . Int J Surg Case Rep. 2019, 63:36-9. 10.1016/j.ijscr.2019.09.015

14. Achebe JU, Njeze GE, Okwesili OR: Treatment of unilateral giant fibroadenoma by breast reduction skin incision: the inverted "T" technique. Niger J Clin Pract. 2014, 17:43-6. 10.4103/1119-3077.122835

15. Ciftci I, Sekmenli T, Ozbek S, Karamese M, Ugras S: Inframammarial giant fibroadenoma removing and a nipple-sparing breast reconstruction in an adolescent: a case report. Prague Med Rep. 2015, 116:161-6. 10.14712/23362936.2015.54

16. Singh G, Kohli PS, Bagaria D: Lateral Oncoplastic Breast Surgery (LOBS) - a new surgical technique and short term results. Am J Surg. 2018, 216:1166-70. 10.1016/j.amjsurg.2017.09.016

17. Preoperative antibiotics and surgical site infection in breast surgery. (2017). Accessed: May 12, 2021: https://www.breastsurgeons.org/docs/statements/Consensus-Guideline-on-Preoperative-Antibiotics-andSurgical-Site-Infe.... 